\title{
Multimodal Interference Based on Large-Core Air-Clad Photonic Crystal fibres for Simultaneous Measurement of Multiparameters
}

\author{
Susana Silva $^{\text {*a,b }}$, J. L. Santos ${ }^{\mathrm{a}, \mathrm{b}}$, F. X. Malcata ${ }^{\mathrm{c}, \mathrm{d}}$, Jens Kobelke ${ }^{\mathrm{c}}$, Kay Schuster ${ }^{\mathrm{c}}$, O. Frazão ${ }^{\mathrm{a}}$ \\ a INESC Porto, Rua do Campo Alegre 687, 4169-007 Porto, Portugal \\ b Departamento de Física da Faculdade de Ciências da Universidade do Porto, Rua do Campo Alegre 687, 4169-007 Porto, \\ Portugal \\ ${ }^{\mathrm{c}}$ ISMAI - Instituto Superior da Maia, Avenida Carlos Oliveira Campos, 4475-690 Avioso S. Pedro, Portugal \\ ${ }^{d}$ CIIMAR - Centro Interdisciplinar de Investigação Marinha e Ambiental, Rua dos Bragas 289, 4050-123 Porto, Portugal \\ ${ }^{\mathrm{e}}$ Institute for Physical High Technology Jena, Albert-Einstein-Str. 9, 07745 Jena, Germany
}

\begin{abstract}
This work describes a large-core air-clad photonic crystal fibre-based sensing structure that is sensitive to refractive index, temperature and strain. The sensing head is based on multimodal interference, and relies on a single mode - largecore air-clad photonic crystal fibre - single mode fibre configuration. Using two distinct large-core air-clad PCF geometries it is possible to obtain an optical spectrum with two dominant loss bands, at wavelengths that have different sensitivities to physical parameters. This characteristic is explored to demonstrate a sensing head that permits the straintemperature discrimination functionality. It is also shown the large-core air-clad photonic crystal fibre can be applied to implement a sensing head sensitive to the water refractive index changes induced by temperature variations.
\end{abstract}

Keywords: Photonic crystal fibre, multimode interference, simultaneous measurement, refractive index.

\section{INTRODUCTION}

In the past few years, multimode interference in optical fibre structures has been studied towards the development of new optical devices [1-2]. These fibre optic devices are usually based on a single mode - multimode - single mode fibre structure, and present unique spectral characteristics that make them suitable for optical communications and sensing [3,4]. For example, Wang et al reported a configuration of this type where by applying a numerical beam propagation method it was determined an optimum value for the length of the multimode fibre section that turns feasible the fibre structure to operate as a refractometric sensor [5].

The development of air-silica microstructured fibres for single-mode operation have gained strong attention due to their novel waveguide properties that made them suitable for a large field of applications, ranging from non-linear effects to optical sensing [6]. Nowadays, research interests are being expanded into large-core air-clad photonic crystal fibres (PCFs) [7] due to their multimode propagation characteristics and their large potential for laser applications [8].

In this work, the experimental results of a large-core air-clad PCF-based sensing structure are presented. The proposed sensing device relies on two distinct in-line air-clad PCFs and is interrogated in transmission. The optical spectrum presents two resonant wavelength loss peaks that are sensitive to refractive index, temperature and strain. It is shown this configuration allows temperature and strain discrimination. Also, a refractometric sensor using this type of fibre is demonstrated.

\section{EXPERIMENTAL RESULTS}

Photonic crystal fibres are generally pure silica fibres where the refractive index contrast required to ensure guidance of light arises by the presence of air-holes, with a specific geometric size and arrangement, rather than by using doped glasses with different refractive indices. This is an important feature, especially for large-core PCFs that must have a small index step, which can be controlled only by the configuration of air-holes.

The working basis of a large-core air-clad PCF section spliced between single-mode fibres relies on the multimode interference principle. When the light field propagating along the input SMF enters the air-clad PCF section, high-order

* sfsilva@inescporto.pt; phone: (+351) 220402 301; fax: (+351) 220402437

21st International Conference on Optical Fiber Sensors, edited by Wojtek J. Bock, Jacques Albert, Xiaoyi Bao, Proc. of SPIE Vol. 7753, 77534A · C 2011 SPIE · CCC code: 0277-786X/11/\$18 · doi: 10.1117/12.885999 
modes are excited so interference between different modes occurs. Light is then coupled into the output SMF where the coupling efficiency, for a given length $L$ of the air-clad PCF section, is wavelength-dependent. Also, the optical power coupled out to the SMF will depend on the amplitudes and relative phases of the various modes of the air-clad PCF at its output end.

To demonstrate the proposed configuration, the sensing head structure shown in Figure 1a) was implemented. It consists of two in-line sections of large-core air-clad PCFs, spliced between SMF-28 single-mode fibres and interrogated in transmission. In this simple approach, one used a broadband source in the $1550 \mathrm{~nm}$ spectral range with a bandwidth of $100 \mathrm{~nm}$, and an optical spectrum analyzer as the interrogation unit. The detail of each PCF implemented in the experiment is shown in Figure 1b).

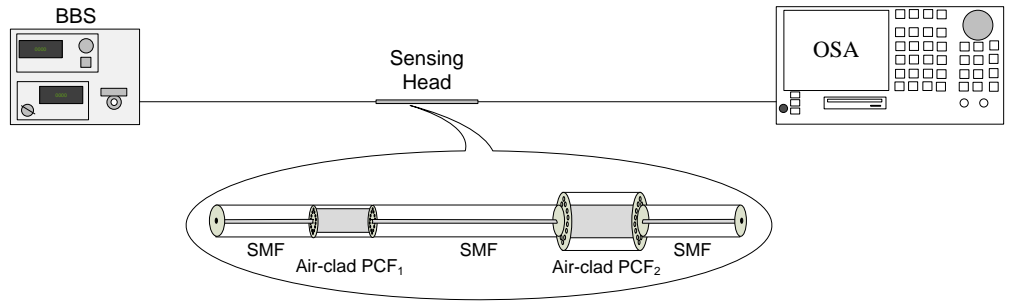

(a)

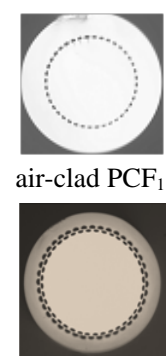

air-clad $\mathrm{PCF}_{2}$

(b)

Figure 1. a) Experimental setup of the air-clad PCF-based sensing structure, and b) detail of each air-clad PCF.

Two air-clad PCFs with different dimensions were used: the air-clad PCF $_{1}$ had $79 \mu \mathrm{m}$-core and $127 \mu \mathrm{m}$-cladding diameters where the silica core was surrounded by a ring of air holes with a ratio of hole diameter, $d$, to pitch, $\Lambda, d / \Lambda=$ 0.5; the air-clad $\mathrm{PCF}_{2}$ had $129.8 \mu \mathrm{m}$-core and $200.7 \mu \mathrm{m}$-cladding diameters and the silica core was surrounded by two rings of air holes, each one with a 0.54 and $0.2 d / \Lambda$ ratios. The optimized length found, for each air-clad PCF section, was $4.2 \mathrm{~cm}$ and $4.3 \mathrm{~cm}$, for air-clad $\mathrm{PCF}_{1}$ and air-clad $\mathrm{PCF}_{2}$, respectively. The distance between air-clad PCFs is approximately $2 \mathrm{~m}$, in order to eliminate modal interference between fibres. Each air-clad PCF was characterized individually and the corresponding optical spectra are shown in Figure 2a); the transmission spectrum of the proposed sensing structure, where the two air-clad PCF are in-line and spliced between SMF, is depicted in Figure 2b).
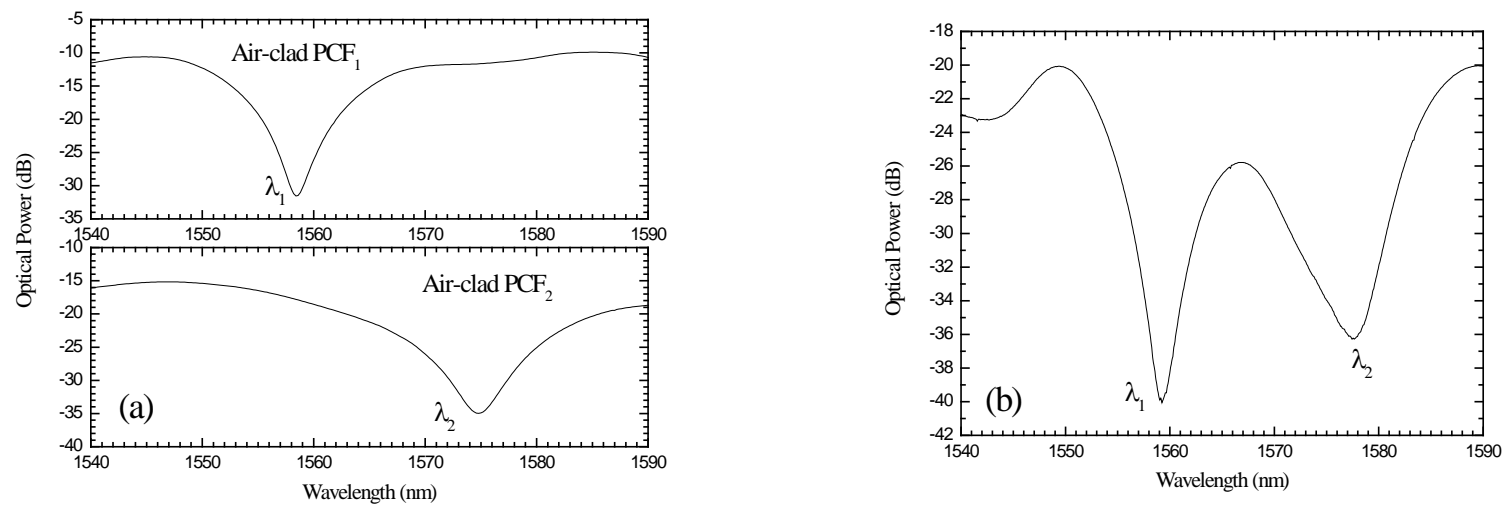

Figure 2. Optical spectra of a) air-clad $\mathrm{PCF}_{1}$, air-clad $\mathrm{PCF}_{2}$ and b) air-clad PCF-based sensing structure.

It can be observed that, in such configuration, the optical power transmitted presents two wavelength loss bands (centred at $\lambda_{1}$ and $\lambda_{2}$ ) - which, in principle, will be sensitive to different physical parameters, viz. refractive index, temperature and strain.

The response to temperature variations of the sensing head was characterized, as shown in Figure 3 . The structure was placed in a tube furnace, and submitted to increasing values of temperature in the range $[0-100]^{\circ} \mathrm{C}$, with $10{ }^{\circ} \mathrm{C}$-steps. The results indicate that the loss bands centred at $\lambda_{1}$ and $\lambda_{2}$ have linear response to temperature variations, characterized by similar sensitivities: $(10.46 \pm 0.01) \mathrm{pm} /{ }^{\circ} \mathrm{C}$ and $(11.15 \pm 0.01) \mathrm{pm} /{ }^{\circ} \mathrm{C}$ for $\lambda_{1}$ and $\lambda_{2}$, respectively. This means that the 
temperature variation does not change the single mode - air-clad PCF launching conditions. Instead, the length variation of both air-clad PCFs (due to thermal expansion) and the air-clad PCF core refractive index variation (due to silica thermo-optic effect) only change the optical path length, causing the wavelength shift of each resonance peak.



Figure 3. Temperature response of the two loss bands centred at $\lambda_{1}$ and $\lambda_{2}$, for the air-clad PCF-based sensing structure.

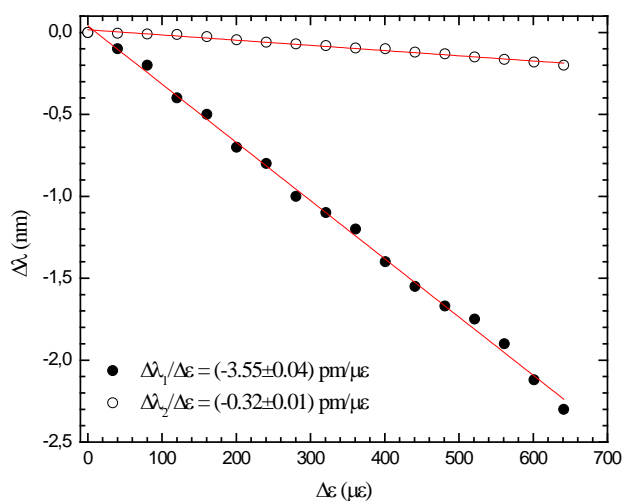

Figure 4. Strain response of the two loss bands centred at $\lambda_{1}$ and $\lambda_{2}$, for the air-clad PCF-based sensing structure.

The sensing structure was also characterized in terms of strain. The input and output fibres were accordingly fixed at two points $2.5 \mathrm{~m}$ apart, and submitted to specific strain values by using a translation stage (via successive $100 \mu \mathrm{m}$ displacements). The results are made available in Figure 4.

Once again, the resonances have linear responses to strain variations, but with different sensitivities: $(-3.55 \pm 0.04)$ $\mathrm{pm} / \mu \varepsilon$ and $(-0.32 \pm 0.01) \mathrm{pm} / \mu \varepsilon$, for $\lambda_{1}$ and $\lambda_{2}$, respectively. This result was somewhat expected since the response to strain of the proposed sensing structure is highly dependent on the cross-section area of each air-clad PCF. Due to that, the resonance loss peak, $\lambda_{2}$, associated to the air-clad $\mathrm{PCF}_{2}$, presents almost no sensitivity to strain due to its large geometrical dimensions. Therefore, the proposed sensing structure can work out as a strain-temperature discrimination sensor. The relationship between wavelength shifts $\Delta \lambda_{1}$ (air-clad $\mathrm{PCF}_{1}$ ) and $\Delta \lambda_{2}$ (air-clad $\mathrm{PCF}_{2}$ ) induced by changes in temperature $(\Delta \mathrm{T})$ and strain $(\Delta \varepsilon)$, may be expressed in a matrix form so that the values for the measurands can be obtained from:

$$
\left[\begin{array}{c}
\Delta \mathrm{T} \\
\Delta \varepsilon
\end{array}\right]=\frac{1}{36.23}\left[\begin{array}{cc}
0.32 & 3.55 \\
-11.15 & 10.46
\end{array}\right]\left[\begin{array}{l}
\Delta \lambda_{1} \\
\Delta \lambda_{2}
\end{array}\right]
$$

When the sensing structure was placed in water and heated it was observed that the shift behaviour of the two loss bands (Figure 2b) was different, as shown in Figure 5, in contrary to what happened when the sensing head was heated in air (Figure 3). In view of the dimensions of the air-clad $\mathrm{PCF}_{2}$, the water infiltrates along the air holes changing the refractive index of this region that behaves as the cladding of the fibre and, therefore, the effective index of the modes guided in the core. This does not happen with air-clad $\mathrm{PCF}_{1}$ because the air holes in the splice zone are obstructed. Consequently, the different behaviour can be connected with the presence of water in the holes and with the circumstance the temperature induced refractive index variation of water is orders of magnitude higher that of air.

This result opened the possibility to use the sensing head of Figure 1a) as a refractometer, in the present case to detect the temperature induced water refractive index variations. The air-clad $\mathrm{PCF}_{1}$ section was used as the temperaturecompensation element. The second section (air-clad $\mathrm{PCF}_{2}$ ) was sensitive to both physical parameters (temperature and refractive index of the surrounding medium). Therefore, in the wavelength shift of the loss band associated with the airclad $\mathrm{PCF}_{2}$ one can remove the temperature component. In first order, the temperature influence comes only from the temperature-induced change of the liquid refractive index, the thermo-optic coefficient of distilled water being $\sim 1 \times 10^{-4}$ $\mathrm{K}^{-1}$ [9]. Figure 5b) presents the wavelength shift dependence with refractive index of water obtained from Figure 5a) after removing the direct temperature effect and using the calibration factor that is the slope of the linear dependence water refractive index variation versus temperature (inset of Figure 5b). 

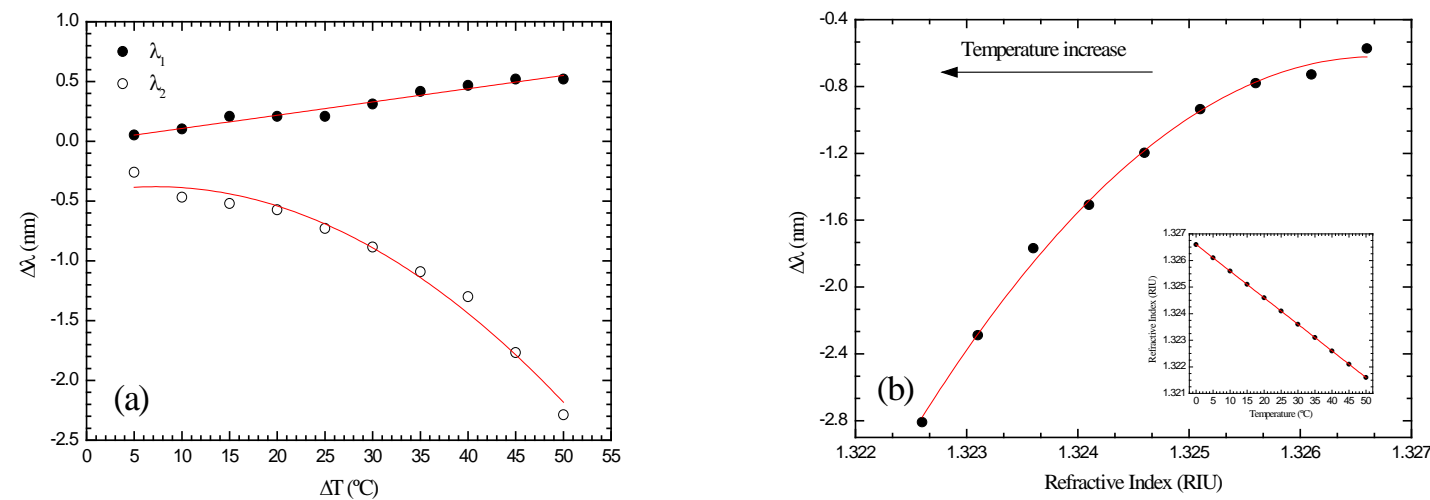

Figure 5. a) Wavelength shift of the sensing head to temperature variations of water and b) refractive index response.

The results shown in Figure 5) indicate a high sensitivity of this sensing head to refractive index variations of water, even considering no optimization was attempted - for temperatures below $20^{\circ} \mathrm{C}$ the sensitivity was $\sim 230 \mathrm{~nm} / \mathrm{RIU}$ and in the most linear region, a sensitivity of $800 \mathrm{~nm} / \mathrm{RIU}$ was attained. This feature, together with the temperature compensation property, indicates the high potential of this optical fibre sensing structure to be used as a temperature compensated refractometer. In view of this further studies are going one to address issues such as the identification of the origins of the non-linearity observable in Figure 5b), the response time of the sensor (associated with the diffusion velocity of the liquids into the $\mathrm{PCF}_{2}$ holes, as well as with the time constant to reach the liquid homogeneity equilibrium inside the holes), and the development of sensing head interrogation techniques not based on the use of the OSA.

\section{CONCLUSIONS}

A large-core air-clad PCF-based sensing structure relying on multimode interference was presented in this communication. The proposed sensing device is based on two distinct in-line air-clad PCFs and interrogated in transmission. The optical spectrum presents two narrow spectral loss bands - which shift with similar sensitivities to temperature but different sensitivities to strain. This feature allowed discriminating both physical parameters. Also, it was identified this sensing head can work as a temperature compensated refractometer.

\section{REFERENCES}

1. W. S. Mohammed, P. W. E. Smith, X. Gu, “All-fibre multimode interference bandpass filter”, Opt. Lett., 31, 25472549 (2006).

2. O. Frazão, J. Viegas, P. Caldas, J. L. Santos, F. M. Araújo, L. A. Ferreira, F. Farahi, “All-fiber Mach-Zehnder curvature sensor based on multimode interference combined with a long-period grating”, Opt. Lett, 32, 3074-3076 (2007).

3. A. Kumar, R. K. Varshney, C. S. Antony, P. Sharma, "Transmission characteristics of SMS fiber optic sensor structures”, Opt. Communications, 219, 215-219, (2003).

4. Q. Wang, G. Farrell, W. Yan, "Investigation on Single-Mode - Multimode - Single-Mode Fiber Structure", J. Lightwave Technol., 26, 512-519 (2008).

5. Q. Wang, G. Farrell, "All-fibre multimode-interference based refractometer sensor: proposal and design”, Opt. Lett., 31, 317-319 (2006).

6. O. Frazão, J. L. Santos, F. M. Araújo, L. A. Ferreira, “Optical sensing with photonic crystal fibers”, Laser and Photonics Reviews, 2, 449-459 (2008).

7. K. Schuster, V. Reichel, J. Kobelke, A. Schwuchow, J. Kirchhof, A. B. Wojcik, "Effects of optical power and thermal impacts on microstructured and low index coated fibers - a comparison", Proc. 55th IWCS 2006, Providence, Transactions of the International Wire and Cable Symposium, 480-483 (2006).

8. J. Kobelke, S. Unger, K. Schuster, A. Schwuchow, J. Kirchhof, "Recent developments of microstructured fibers for active and passive applications”, PHOTONICS-2008: International Conference on Fiber Optics and Photonics (2008).

9. G. Abbate, U. Bernini, E. Ragozzino, and F. Somma, The temperature dependence of the refractive index of water, Journal Physics. D: Applied Physics, 16, 1606-1612 (1998). 